\title{
Rapid diagnostic tests used in first-step health services?
}

\author{
Huseyin Guducuoglu ${ }^{1}$
}

\section{Dear Editor,}

The term 'point-of-care test' (POCT) has been previously defined as a rapid diagnostic assay. The term has now been expanded to include any test that can be performed rapidly outside of a central laboratory environment (including home testing) even when the result might not directly impact on patient outcome (1). In recent years increased investment, technological advances, and greater awareness about the importance of reliable diagnostic tests has resulted in rapid progress. Rapid, reliable and affordable POC tests are requiring no equipment and minimal training (2).

\section{Some rapid microbiological tests:}

Pneumococci: The diagnostic standard is still sputum or blood culture and the Gram stain. A pneumococcal rapid test can be used to increase diagnostic yield. A negative test does not reliably exclude pneumococcal pneumonia (sensitivity: $50 \%$ to $80 \%$; specificity: 90\%).

Legionella: Legionella testing is appropriate in all unclear cases of pneumonia. A test is recommended each patient with pneumonia of unclear origin after admission to an intensive care ward, in epidemics, and when beta-lactam therapy fails. The diagnostic method of choice is antigen detection in the urine (sensitivity of $94 \%$, specificity $99 \%$ to $100 \%$ ).

Influenza: There should be no routine testing for influenza antigens. This may be helpful in outbreaks or before the decision to start antiviral therapy. A test should be used which can differentiate between influenza types A and B (sensitivity $50 \%$ to $96 \%$ and specificity $72 \%$ to $100 \%$ ).

S. pyogenes: The rapid test for group A streptococci is now established as a routine component of diagnosis. Specific use markedly reduces unnecessary antibiotic use [sensitivity $>85 \%$, specificity ( $>95 \%$; cf. culture: sensitivity $80 \%$ to $97 \%$, specificity $100 \%$ )].

S. agalactiae: The rapid test for group B streptococci is currently not sensitive enough to replace detection in culture. Routine use is not recommended [sensitivity ( $11 \%$ to $79 \%$; cf. culture: $91 \%)$, specificity (91\% to $100 \%$; cf. culture: $89 \%$ )].

HIV: The rapid test for HIV has been fully developed in diagnosis and is just as reliable as conventional screening diagnosis with EIA. It can be used for patients who are difficult to reach, in regions with poor laboratory access, and in urgent decisions on possible prophylaxis after exposure or transmission [sensitivity $(98 \%$ to $100 \%)$ and specificity $(86 \%$ to $100 \%$, one outlier $75 \%$ ), even though the specificity is $99 \%$ to $100 \%$ in some studies]

Malaria (P. falciparum): The rapid test is now a very good alternative to light microscopy, although it has not replaced this as "gold standard". It can be used when light microscopy is not available. The rapid test has failed in isolated cases in spite of high parasitemia [sensitivity of the tests is usually over $90 \%$ and the specificity over $80 \%$ ] (3).

The health system in Turkey can be divided into three groups as noted in the article (4); First-step health services (Family health center): The health system that can reach the individual and the families within the society as a whole, solve health problems of the community, protect the health, and provide home and outpatient therapy services. Second-step health services: The services provided for diagnosis and inpatient therapy of the patients. Third-step health services: Health services provided for the diseases requiring advanced examination and special therapy (3). In the provision of health services, the term "quality" may be defined as "diagnosis, therapy and care services. In several countries, a first-step physician who has received postgraduate training according to the international standards is designated as a "General Practitioner". A family physician will be required, apply actual screening, protect, provide therapy and follow-up protocols, and carry out multidisciplinary researches required by the first step (4). This physician must implement some simple tests in the first-step health service laboratory for diagnosis.

First-step health services are acting as primary health care services that use in-house microbiological rapid diagnostic tests (fast, reliable, simple) in the laboratory. This tests used in such health care facilities are limited and low applicability. Sensitivity and specificity of some of the rapid diagnostic tests (As seen above) are high. Such tests can be used easily in primary health care. If we give examples of them, Streptococcus pyogenes rapid test used for diagnosis of Group A Streptococcus (GAS), we can be easily avoided, likely to be carditis and accordingly complications in the future (in the diagnosis omitted). The cost of tests is extremely low. These tests are applicable in a very short time. Therefore, if rapid diagnostic tests being used in such institutions, many diseases can be easily eradicated with treatment and diagnosis can be made. 


\section{References}

1. Moore C. Point-of-care tests for infection control: should rapid testing be in the laboratory or at the front line? J Hosp Infect. 2013; 85(1): 1-7.

2. Peeling RW, Mabey D. Point-of-care tests for diagnosing infections in the developing worl. Clin Microbiol Infect. 2010;16(8):1062-9
3. Stürenburg E, Junker R. Point-of-care testing in microbiology: the advantages and disadvantages of immunochromatographic test strips. Dtsch Arztebl Int. 2009;106(4):48-54

4. Bulut A, Oguzoncul AF. Evaluating the level of satisfaction of patients utilizing first-level health facilities as a function of health system performance rating in the province of Elazig, Turkey. Patient Prefer Adherence. 2014; 28(8):1483-92.

Copyright (C) 2014 The Author(s); This is an open-access article distributed under the terms of the Creative Commons Attribution License (http://creativecommons.org/licenses/by/4.0), which permits unrestricted use, distribution, and reproduction in any medium, provided the original work is properly cited. 